\title{
Modelling of simultaneously obtained small and wide angle synchrotron-radiation scattering depth profiles of ordered titania nanotube thin films
}

\author{
Krunoslav Juraić a, *, Milivoj Plodinec ${ }^{\text {a }}$, Irena Kereković ${ }^{b}$, Daniel Meljanac ${ }^{a}$, Vilko Mandić ${ }^{c}$, Davor Gracin ${ }^{\text {a }}$, \\ Vesna Janicki ${ }^{a}$, Sigrid Bernstorff ${ }^{\mathrm{d}}$, Miran Čeh ${ }^{\mathrm{e}}$, Aden Hodzic ${ }^{\mathrm{f}}$, Andreja Gajović ${ }^{\mathrm{a}}$ \\ ${ }^{a}$ Ruđer Bošković Institute, Bijenička cesta 54, Zagreb, Croatia \\ ${ }^{\mathrm{b}}$ University of Applied Health Sciences, Mlinarska cesta 38, 10000, Zagreb, Croatia \\ ${ }^{\mathrm{c}}$ Faculty of Chemical Engineering and Technology, Marulicév trg 19, Zagreb, Croatia \\ d Sincrotrone Trieste, Strada Statale 14, km 163.5, 34149, Basovizza, TS, Italy \\ e Jozef Stefan Institute, Ljubljana, Jamova 11, Slovenia \\ ${ }^{f}$ Central European Research Infrastructure Consortium, Strada Statale 14, km 163.5, 34149, Basovizza, TS, Italy
}

\section{ART ICLE INFO}

Keywords:

Nanotube array

Titanium dioxide

GISAXS

GIWAXS

Anodization

\begin{abstract}
A B S T R A C T
Thin-films of vertically aligned titanium dioxide nanotubes are key constituents of charge transfer layers in 3rd generation photovoltaics. The beneficial charge transport primarily occurs due to the favourable microstructural features, i.e. the large effective surface-to-volume ratio. Here measuring methods are selected which are sensitive to microstructural features: Simultaneous Grazing Incidence Small and Wide Angle X-Ray Scattering (GISWAXS) and Scanning Electron Microscopy (SEM). Further, a model is developed for the simulation and explanation of the GISWAXS experimental data. Titanium nanotube arrays were investigated, which were previously successfully produced by electrochemical anodization of titanium thin film evaporated onto a zinc-oxide coated glass substrate. The developed model was shown to be appropriate to describe the obtained samples, which consist of arrays of single nanotubes with a diameter of $40-80 \mathrm{~nm}$, and a titanium dioxide layer with a porosity of 30-50\%. Within this work, we presented simultaneous GISWAXS as a comparable tool to SEM for the fast and successful study of the average nanotubes' diameters in nanotubes arrays.
\end{abstract}

\section{Introduction}

Titanium dioxide $\left(\mathrm{TiO}_{2}\right)$ is one of the most intensively investigated compounds in material science due to its favourable essential properties. It is a wide band gap semiconductor having band-edge positions appropriate for solar cell applications and for hydrogen generation by water splitting $[1,2]$. It is also known as a non-toxic, environment-friendly and corrosion-resistant material. Nano-forms of $\mathrm{TiO}_{2}$ such as nanoparticles, nanorods, nanowires and, in particular, nanotubes can be used as photo-electrodes in dye-sensitized solar cells $[3,4]$. The conversion efficiency of solar cells can be significantly improved by using oriented nanorod-like materials on top of a transparent conductive oxide [5].

Vertically aligned $\mathrm{TiO}_{2}$ nanotubes arrays can be prepared by electrochemical anodization of titanium foil in a fluoride electrolyte. Over the last decade, significant progress has been made in this field, particularly to obtain and to optimize the morphology of highly ordered $\mathrm{TiO}_{2}$ nanotube arrays [6-8].
In this work, instead of using a several hundred microns-thick titanium foil as substrate for anodization, titanium thin films were used deposited directly onto transparent conductive oxide (TCO) coated glass substrates. The successful preparation of nanotube arrays in the form of transparent thin films on TCO/glass provides the opportunity for their application in thin films solar cells (perovskite or dye-sensitized). In addition, the same experimental procedure can be used for a straightforward preparation of such arrays on various other substrates, which could be beneficial in a range of applications.

Microscopic techniques like scanning electron microscopy (SEM) and high-resolution transmission electron microscopy (HRTEM) are usually used for structural composition and surface morphology analysis of such samples. While SEM and HRTEM yield information about materials in direct space (which can be easily analysed), there are complementary techniques such as X-ray diffraction and scattering techniques which give information in the reciprocal space. Among this, grazing incidence X-ray diffraction (GIXRD) and small angle X-ray scattering (GISAXS) are particularly suitable for thin film sample analysis. These grazing incidence techniques are relatively new and

\footnotetext{
* Corresponding author.

Email address: kjuraic@irb.hr (K. Juraić)
} 
therefore there are only a few publications related to the investigation of ordered nanotube array samples performed by GISAXS. In this sense, Neelima [9] recently compared GISAXS and neutron scattering results obtained from titania nanotubes arrays.

GISWAXS is an experimental technique which gives insight into the material crystal structure (GIWAXS) and the surface layers morphology on the nanometer scale (GISAXS). The use of synchrotron $\mathrm{X}$-ray radiation (high flux, brilliance and stability) allows to perform GISWAXS even on very thin films or small sample volumes, and on very weakly scattering materials. Two main advantages of GISWAXS over microscopy are, that data are obtained averaged over a "large area" (several square millimetres), and that simultaneously information can be obtained about the crystal structure and surface morphology on the nanometer scale. By varying the grazing incidence angle, the penetration depth of the probing X-ray beam can be changed from the surface layer towards the bulk of the sample, which enables to perform a depth-dependent analysis across the sample. When comparing SEM and GISAXS, the main disadvantage of the latter one is the need for a proper computational model that for analysis the data interpretation proper fitting models for the experimental data are needed and this work is, in its most important part, a contribution to overcoming this problem.

Recently the preparation of $\mathrm{TiO}_{2}$ nanotube array thin films and the influence of the Ti substrate morphology on the nanotubes' growth has been reported [10]. In this work, the focus lies on the quantitative analysis of the nanotubes dimensions (inner radius and wall thickness) obtained by GISWAXS. Most part of this work is devoted to developing an appropriate way for the analysis of the GISAXS data for the actual case, nanotube arrays. A model is outlined for the analysis of GISAXS intensity pattern obtained on such samples, which is based on the Distorted-Wave Born Approximation (DWBA) [11] assuming a form factor of vertically aligned core-shell cylinders and including the corrections for the non-perfect geometry of the samples. The simulation results (the nanotubes inner and outer diameter distributions) are then compared with results obtained from SEM micrographs.

\section{Experimental}

\subsection{Sample preparation}

A 3-step process was used for the $\mathrm{TiO}_{2}$ nanotube array thin film samples preparation. In the first step titanium layer was deposited on magnetron sputtered Al-doped zinc-oxide covered glass substrates $20 \mathrm{x}$ $20 \mathrm{~mm}$ in size (Fig. 1a). Prior to the Ti layer deposition, the ZnO-glass substrates were cleaned using the standard procedure in an ultrasonic bath: first $10 \mathrm{~min}$ in acetone, then $10 \mathrm{~min}$ in ethanol and $10 \mathrm{~min}$ in Milli-Q water. Finally, the substrates were dried under flowing nitrogen. The substrates were preheated to $230^{\circ} \mathrm{C}$ and electron beam evaporation was used for the Ti layer deposition (Fig. 1b). The starting material was $99 \%$ Ti $0.2-1.5 \mathrm{~mm}$ granulate from Balzers. The thickness of the deposited Ti layer $(1.2 \mu \mathrm{m})$ was monitored in situ by a quartz crystal microbalance monitor. It is not expected that during this deposition the substrate temperature significantly exceeded $100^{\circ} \mathrm{C}$.

In the second step, the $\mathrm{Ti}$ layers were anodized in the shape of the circular area having $8 \mathrm{~mm}$ in diameter (Fig. 1c) and $\mathrm{TiO}_{2} \mathrm{NTs}$ were formed in that part of $\mathrm{Ti}$ layer as is shown in the schematic view of all the layers in cross section (Fig. 1d). For the anodization at room temperature, a conventional two-electrode system was used with a Pt foil as the counter electrode and a Ti layer as the anode [12]. The diameter of the circular etched area was $10 \mathrm{~mm}$. Ethylene glycol containing $0.3 \mathrm{wt} \%$ of $\mathrm{NH}_{4} \mathrm{~F}$ and $2 \mathrm{vol} \%$ of distilled water was used as electrolyte [12]. The DC voltage for anodization was varied in the range $40-60 \mathrm{~V}$ in order to obtain samples with various nanotube diameter. Since for photovoltaic use, the transparent TiO2 layer is favourable, the anodization time (30-75 min) was adjusted to etch the titanium layer as much as possible and thus to obtain a transparent $\mathrm{TiO}_{2}$ NT layer (Table 1

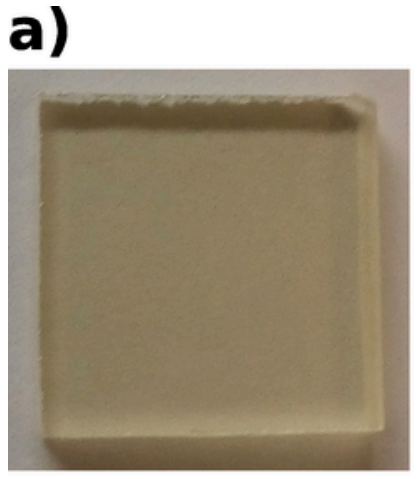

b)
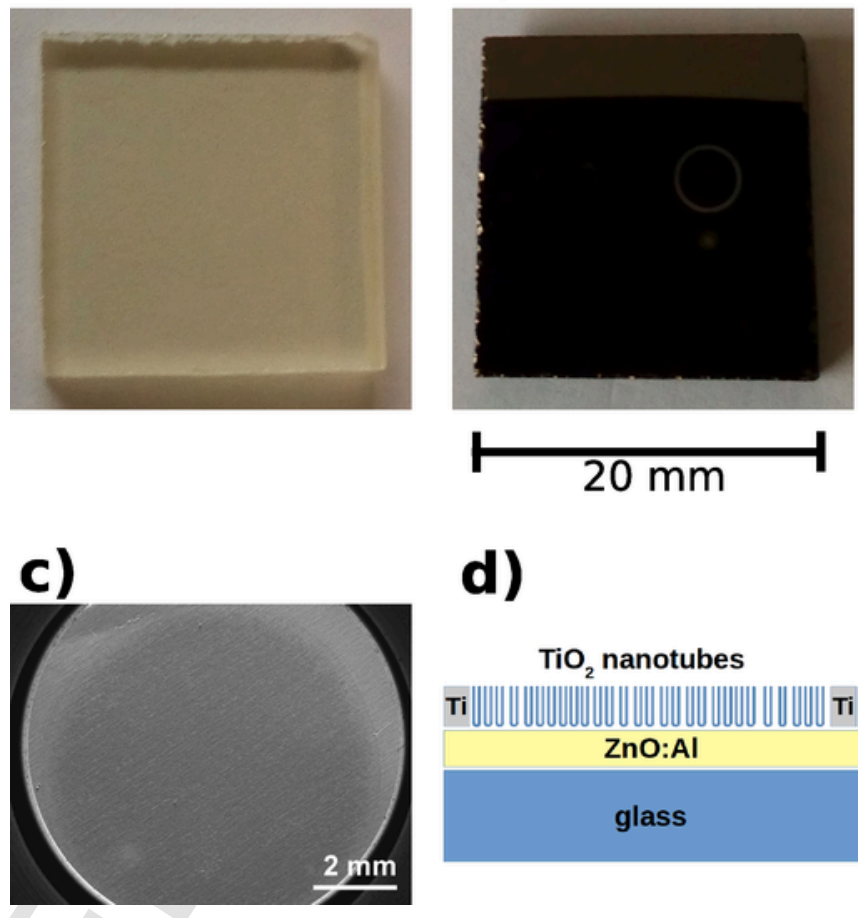

d)

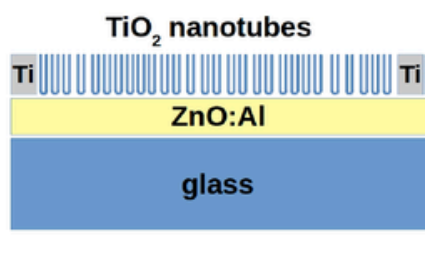

Fig. 1. a) Photo of $\mathrm{ZnO}$ coated glass substrate, b) Photo of deposited Ti thin film on $\mathrm{ZnO}$ coated glass substrate, c) SEM image of Ti sample with circular anodized area containing $\mathrm{TiO}_{2}$ nanotube array (sample NT-60V-60 m) and d) schematic view of $\mathrm{TiO}_{2}$ NT samples cross-section with labelled all layers.

Table 1

Parameters used in anodization process: applied voltage and duration of anodization and results of SEM images analysis: average inner and outer nanotubes radius.

\begin{tabular}{lllll}
\hline Sample & $\begin{array}{l}\text { Anodization } \\
\text { voltage [V] }\end{array}$ & $\begin{array}{l}\text { Anodization } \\
\text { time [min }]\end{array}$ & $\begin{array}{l}\text { SEM R } \\
{[\mathrm{nm}]}\end{array}$ & $\begin{array}{l}\text { SEM R } \\
{[\mathrm{nm}]}\end{array}$ \\
\hline NT-60V-60m & 60 & 60 & $18.0 \pm 2.3$ & $48.4 \pm 5.3$ \\
NT-50V-30m & 50 & 30 & $11.0 \pm 0.9$ & $29.9 \pm 3.0$ \\
NT-40V-75m & 40 & 75 & $8.6 \pm 1.9$ & $33.3 \pm 4.5$ \\
NT-40V-45m & 40 & 45 & $10.1 \pm 1.6$ & $41.9 \pm 3.8$ \\
NT-40V-30m & 40 & 30 & $9.8 \pm 1.8$ & $35.3 \pm 4.3$ \\
\hline
\end{tabular}

). For samples anodized at $40 \mathrm{~V}$, anodization time was varied to obtain layers with different nanotube length.

During the anodization, the applied voltage was kept constant, and the current was monitored by a Fluke 17B multimeter. A typical current variation over time is presented in Fig. 2, so from that variation the phases of NT formation can be recognized. Thus, during the first minute, the current decreases rapidly (phase I), which corresponds to the formation of a compact oxide layer on the electrolyte/Ti interface. Then during the next $3-4$ min current starts to decrease much slower (phase II), that represent the start of nanotubes formation within the Ti. This effect is much more pronounced (with additional plateau) for titanium foil anodization and corresponds to the formation of the porous structure [13]. After that follows a gradual current decrease (phase III) indicating that the formation of pores reach equilibrium with the dissolution, that phase persist up to moment when the titanium layer is completely etched (the electrolyte comes in direct contact with the $\mathrm{ZnO}$ coated glass substrate). At that moment the current starts to increase rapidly. This moment is not presented in Fig. 2, since 


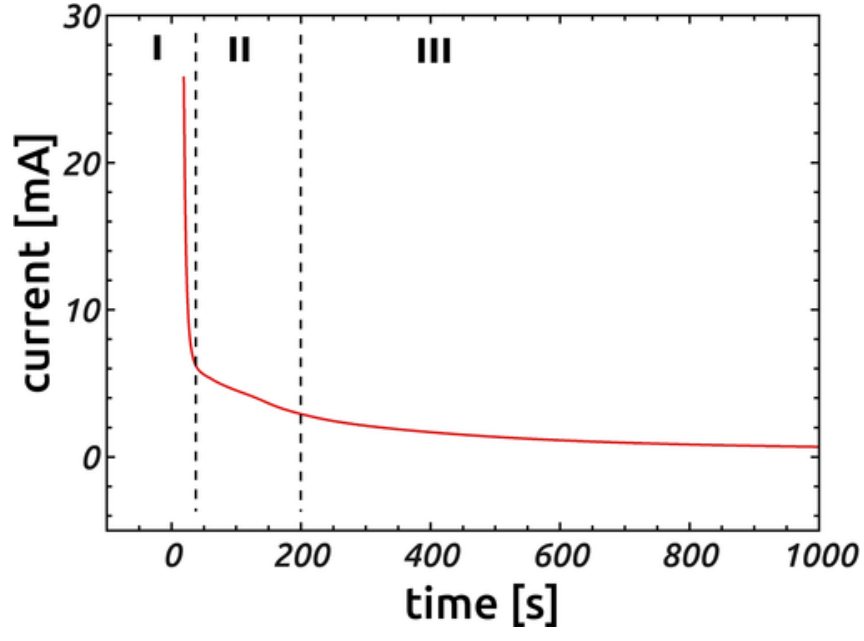

Fig. 2. Time evolution of the anodization current during the anodization process (sample NT-60V-60 m). During each anodization process the applied voltage was kept constant.

the anodization process should be stopped earlier to avoid short circuit in the case of using the prepared materials as electrode transporting layer in dye or perovskite photovoltaics.

After anodization, the formed $\mathrm{TiO}_{2} \mathrm{NT}$ array samples were washed in distilled water and dried by compressed air.

In the third step, the $\mathrm{TiO}_{2} \mathrm{NT}$ array samples were calcined in an oven at $450{ }^{\circ} \mathrm{C}$ for $1 \mathrm{~h}$ in the air atmosphere (heating rate $5^{\circ} \mathrm{C} / \mathrm{min}$ ) in order to obtain $\mathrm{Ti}$ anatase phase.

\subsection{Scanning electron microscopy (SEM) and energy-dispersive X-ray spectroscopy (EDXS)}

The surface morphology and chemical analyses of the sample were obtained with a field-emission-gun (FEG) scanning electron microscope (JEOL JSM-7610F). The imaging was performed at $10 \mathrm{kV}$ accelerating voltage and a working distance of $8 \mathrm{~mm}$ with the SEI detector configuration. For energy-dispersive X-ray spectroscopy (EDXS), INCA Oxford 350 EDS SDD was used. Spectra were recorded at $15 \mathrm{kV}$ accelerating voltage and $15 \mathrm{~mm}$ working distance.

Several SEM images obtained at higher magnification (X 100k) were used for the quantitative analysis. Using the ImageJ software, the areas of each nanotube (inner and outer) were marked. The diameters of each marked nanotube were calculated from the obtained areas assuming that the nanotubes have a perfect circular cross-section.

\subsection{Grazing incidence small- and wide-angle X-Ray scattering (GISWAXS)}

Simultaneous GISWAXS experiments were performed at the Austrian SAXS beamline at the Elettra synchrotron radiation center in Trieste (Italy) using an $8 \mathrm{keV} \mathrm{X}$-ray beam [14]. The beam size at the sample position was $\mathrm{HxV}=0.2 \mathrm{~mm} \times 1 \mathrm{~mm}(\mathrm{H}=$ normal to sample surface, $\mathrm{V}=$ parallel to sample surface). This beam size was optimized in order to increase the resolution in the direction parallel to the sample surface but at the same time to keep an acceptable signal to noise ratio. For the GISAXS experiment, a Pilatus $1 \mathrm{M}$ detector $(981 \times 1043$ pixels and $172 \times 172 \mu \mathrm{m}^{2}$ pixel size) was positioned in the plane normal to the $\mathrm{X}$-ray beam and at a distance of $1976 \mathrm{~mm}$ from the sample. The samples were measured in air, but a vacuum chamber was used in the space between the sample stage and the GISAXS detector in order to minimize air scattering. The angular positions of the pixels in the GISAXS images were calibrated with the silver behenate powder standard.

The GIWAXS detector (Pilatus 100K, $487 \times 195$ pixels, $172 \times 172 \mu \mathrm{m}^{2}$ pixel size) was positioned very close to the sample at a distance of $177.8 \mathrm{~mm}$ and with a detector to sample surface tilt of $55^{\circ}$ (Fig. 3).

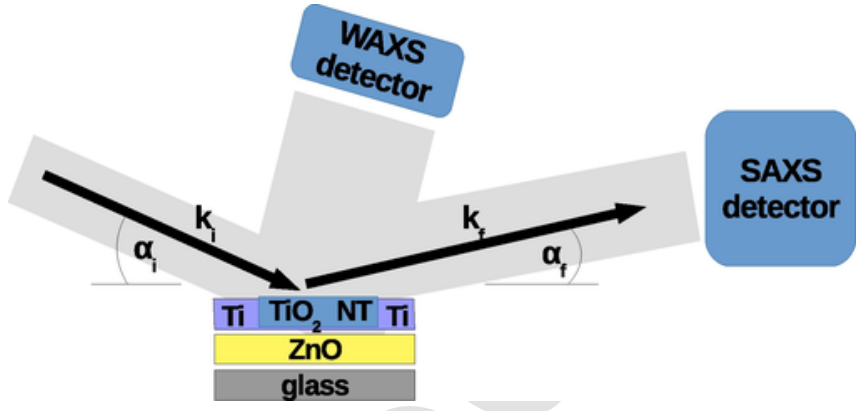

Fig. 3. GISAXS/GIWAXS experimental scheme. The irradiated area of the $\mathrm{TiO}_{2} \mathrm{NT}$ sample is presented and the position of the WAXS and SAXS detectors. The incident wave vector $\mathrm{k}_{\mathrm{i}}$, the scattered wavevector $\mathrm{k}_{\mathrm{f}}$, the angle of incidence $\alpha_{\mathrm{i}}$, and the angle of the scattered beam $\alpha_{\mathrm{f}}$ are indicated.

$\mathrm{LaB}_{6}$ powder (from NIST) in a capillary was used for the GIWAXS angular scale calibration.

The angle of grazing incidence was varied starting from the critical angle $\alpha_{c}$ for total external reflection for the porous $\mathrm{TiO}_{2} \mathrm{NT}$ array and increased in small steps until the $\mathrm{TiO}_{2}-\mathrm{ZnO}$ interface was reached ( $\alpha \mathrm{c}$, $\left.\alpha c+0.1^{\circ}, \alpha c+0.3^{\circ}, \alpha c+0.6^{\circ}, \alpha c+1.0^{\circ}\right)$. The corresponding penetration depth values (calculated for bulk $\mathrm{TiO}_{2}$ ) estimated using equation $\mathrm{A} 8$ in Appendix are: $10 \mathrm{~nm}, 25 \mathrm{~nm}, 165 \mathrm{~nm}, 270 \mathrm{~nm}, 400 \mathrm{~nm}$. The precise values for the grazing incidence angles and especially the critical angle for total external reflection were determined from 1D cuts taken in the specular plane from 2D GISAXS intensity patterns $\left(Q_{y}=0\right)$ detecting the positions of the maximum of the directly reflected beam and the Yoneda maximum.

\section{Results and discussion}

\subsection{SEM and EDXS results}

The surface of the Ti substrate, deposited by electron beam evaporation on $\mathrm{ZnO}$ coated glass and used as the starting layer for anodization, consists of $100-200 \mathrm{~nm}$ large grains. The $30^{\circ}$ tilted SEM image indicates that the larger grains are agglomerates of smaller nanocrystals (Fig. 4f).

The parameters of the anodization process (applied voltage and electrolyte content) were optimized to obtain highly ordered vertically aligned $\mathrm{TiO}_{2}$ nanotube array samples (Fig. 4).

The averaged single nanotube outer diameter measured in SEM images was in the range $60-100 \mathrm{~nm}$ and the inner diameter in range $15-40 \mathrm{~nm}$ (Table 1). Since the outer diameter of the obtained nanotubes was 3-4 times larger than inner, therefore nanotubes have $22-30 \mathrm{~nm}$ thick walls. Usually, obtained wall thickness is much smaller for nanotubes formed by using Ti foil for anodization as was the case in our previous studies [12,15]. From Fig. 5 can be recognized three different classes of samples: 1) samples with very densely packed touching nanotubes (Figs. 5a), 2) samples with almost completely separated nanotubes (Figs. 5b) and 3) samples with nanotubes organized in larger or smaller clusters (Fig. 5f). With the aim to show how the height of the nanotubes varied with anodization time, SEM images of the vertical side of the nanotubes are shown in Fig. 5. Height of prepared $\mathrm{TiO}_{2}$ thin films can be estimated from Fig. 5 where are presented images for samples prepared using identical voltage $(40 \mathrm{~V})$, while anodization time is varied (30, 45 and $75 \mathrm{~min}$ ). Height of the nanotubes obtained after $30 \mathrm{~min}$ of anodization was approximately $500 \mathrm{~nm}$, the nanotubes after $45 \mathrm{~min}$ was $800 \mathrm{~nm}$, while after $75 \mathrm{~min}$ of anodization the height was $1200 \mathrm{~nm}$.

EDXS spectra obtained at every sample were similar, therefore only one representative EDXS are shown in Fig. 6. The spectrum confirms the sample content: the $\mathrm{Ti}$ and $\mathrm{O}$ elements are in the nanotube layer, $\mathrm{Zn}, \mathrm{Al}$ and $\mathrm{O}$ in the $\mathrm{Al}$-doped $\mathrm{ZnO}$ transparent conductive layer and 

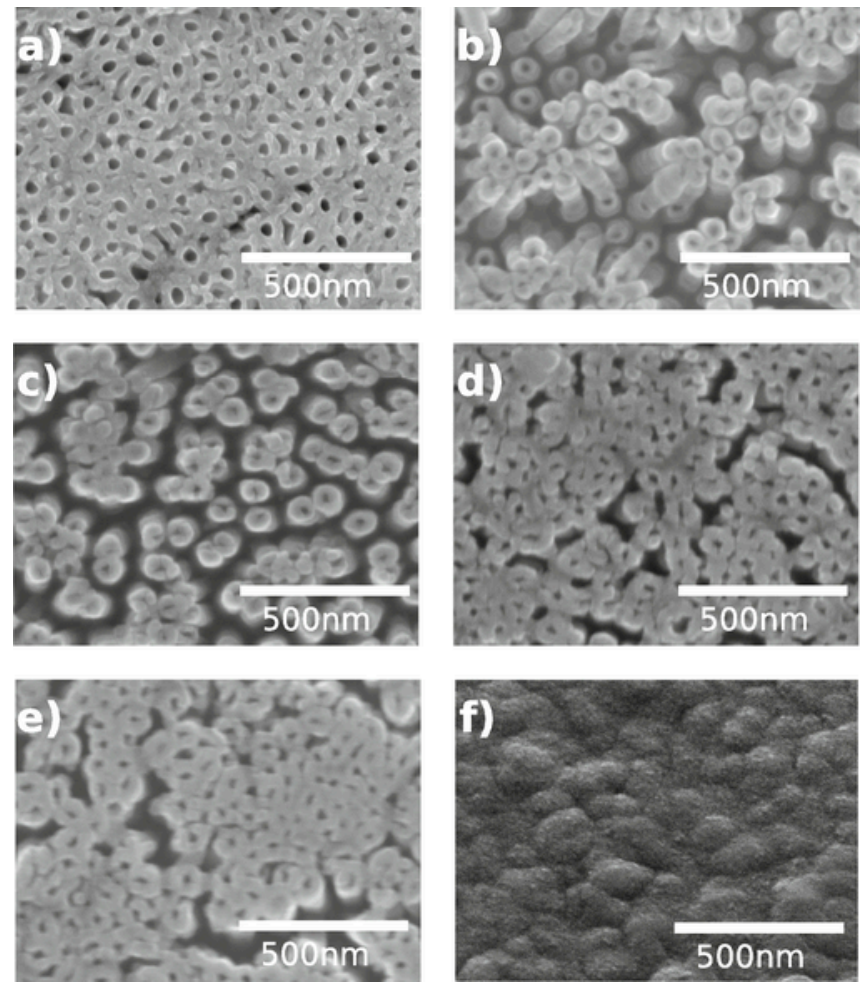

Fig. 4. SEM images of $\mathrm{TiO}_{2}$ nanotube samples obtained under various anodization conditions. a) NT-60V-60 m, b) NT-50V-30 m, c) NT-40V-75 m, d) NT-40V-45m, e) NT-40V-30 m and $\mathrm{f}$ ) an electron beam evaporated Ti layer on a $\mathrm{ZnO}$ :Al coated glass substrate. Sample preparation data and nanotubes diameters corresponding to figures a) - e) are given in in Table 1.

Si in the glass substrate. The EDXS, shown in Fig. $6 \mathrm{~b}$, was taken from the sample area presented in the SEM image of Fig. 6a. The small amount of carbon is related to surface layer contamination.

\subsection{GIWAXS results}

2D GIWAXS patterns (Fig. 7) were reduced to the standard 1D diffraction pattern using the Nika Igor Pro package [16].

The GIWAXS data were used only for qualitative analysis because there is a significant contribution of the instrumental profile to the diffraction peaks broadening due to the used grazing incidence geometry. This is due to the facts that the incident X-ray beam has a very long footprint on the sample surface, and a 2D planar detector (placed close to sample stage) was used for GIWAXS signal detection instead of a point or curved detector. For example, for a grazing angle of incidence of $0.5^{\circ}$ and a beam width of $0.2 \mathrm{~mm}$ in the direction perpendicular to sample surface, the beam footprint on the sample surface is $23 \mathrm{~mm}$ long. Diffraction peak width is highly dependent on sample size. Also, diffraction peak positions are dependent on sample position at the sample stage. This fact can explain small shifts of diffraction peaks from values taken from literature.

Fig. 8 show GIWAXS 1D diffraction patterns for all the studied samples compared to $\mathrm{ZnO}$ coated glass substrate and Ti layer used for anodization. Ti layer, as-deposited onto $\mathrm{ZnO}$ coated glass substrate is completely amorphous (broad peak between $30^{\circ}$ and $45^{\circ}$ ). After anodization samples are still amorphous (60V, $60 \mathrm{~m}$ n.a.), but after the calcination process, the $\mathrm{TiO}_{2}$ anatase phase is formed (appearance of a diffraction peaks characteristic for the anatase phase $\left((101)\right.$ peak at $25^{\circ}$ and other less prominent peaks).
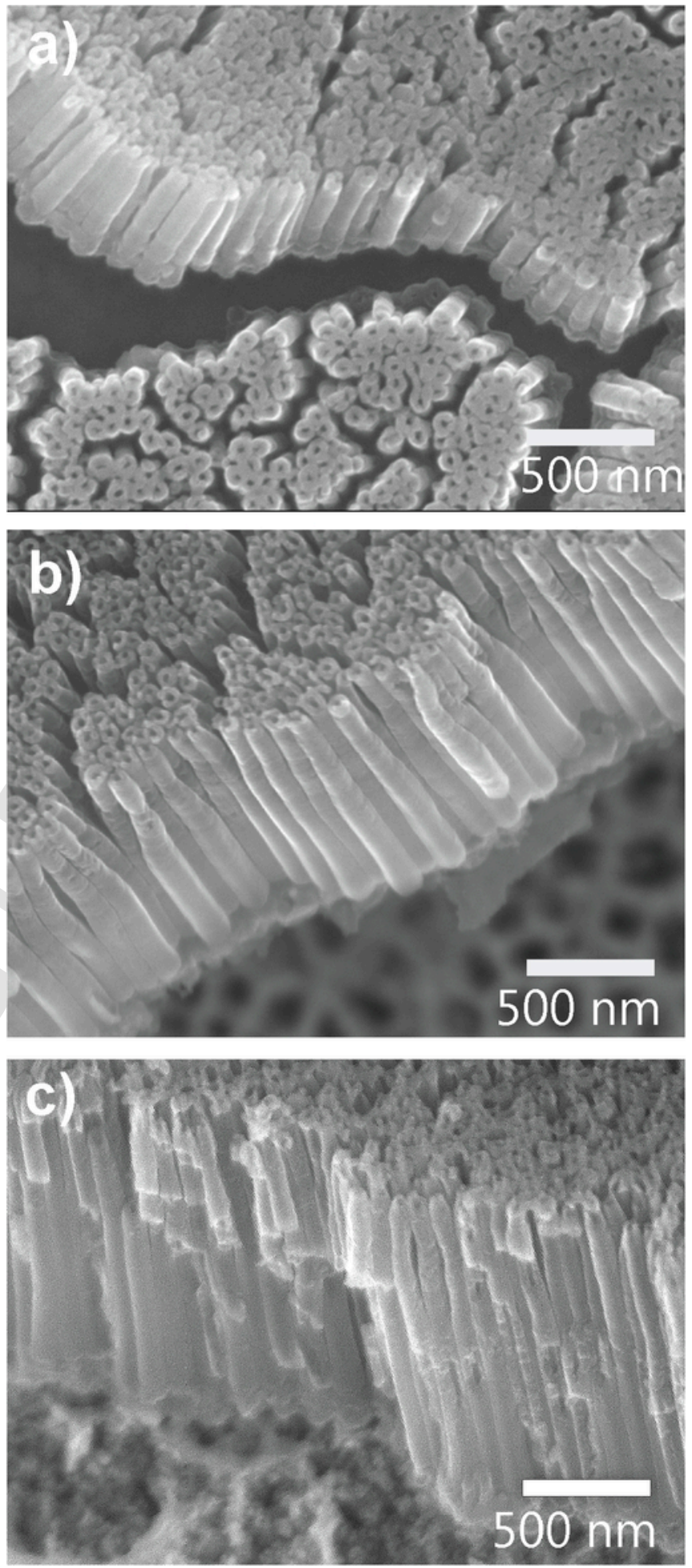

Fig. 5. SEM images of vertical side of nanotubes for samples prepared using identical anodization voltage (40V): a) NT-40V-30 m, b) NT-40V-45 m, c) NT-40V-75 m.

\subsection{GISAXS results}

In Fig. 9 are presented illustration of GISAXS 2D images for as-deposited Ti substrate ( $\mathrm{a}$ and $\mathrm{b}$ ) and $\mathrm{TiO}_{2} \mathrm{NT}$ samples after anodization (c and d). Figures a) and c) are taken for the angle of incidence equal to the critical angle for total external reflection for $\mathrm{Ti}$ and $\mathrm{TiO}_{2} \mathrm{NT}$ 

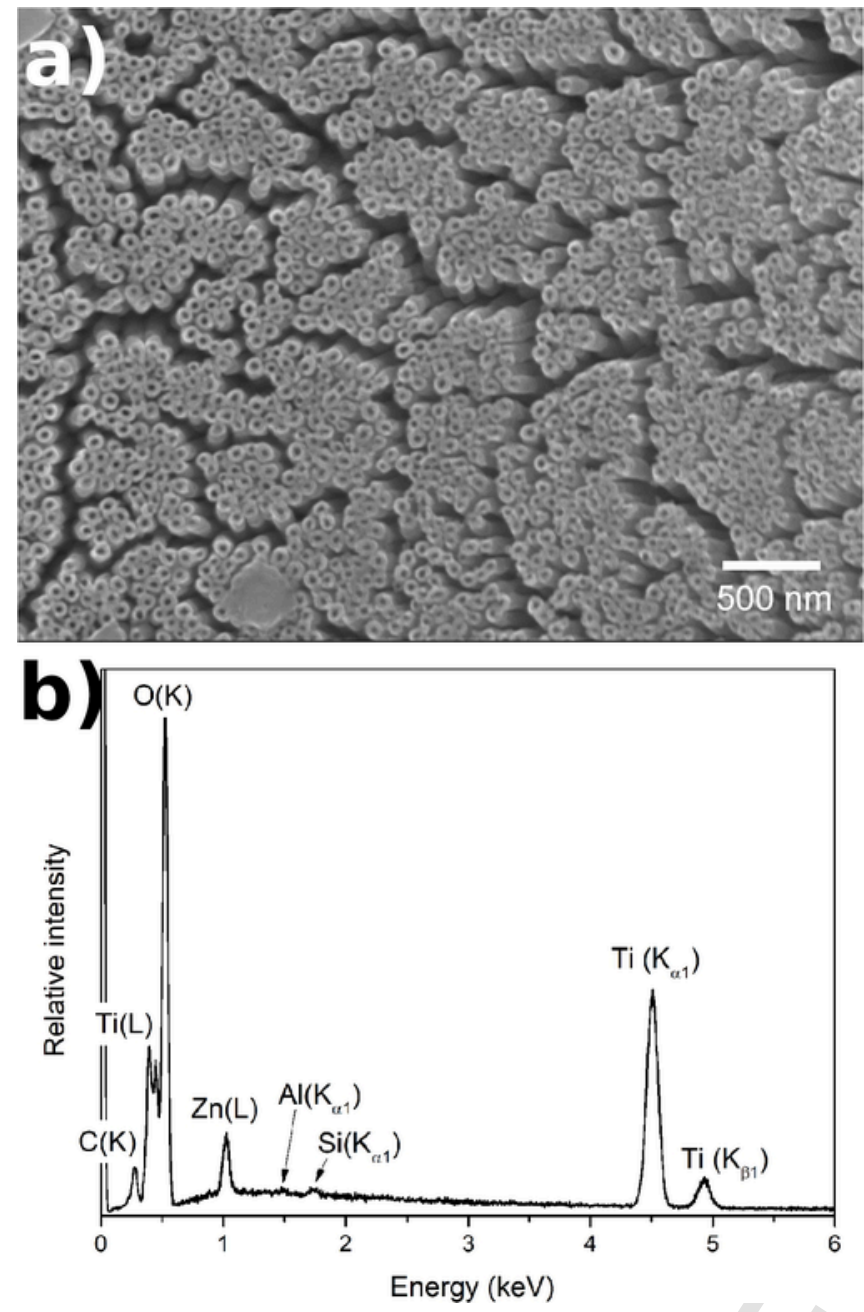

Fig. 6. (a) Area where EDXS was taken and (b) EDXS spectrum of a $\mathrm{TiO}_{2} \mathrm{NT}$ array thin film on a ZnO:Al coated glass substrate (sample NT-40V-45 m).

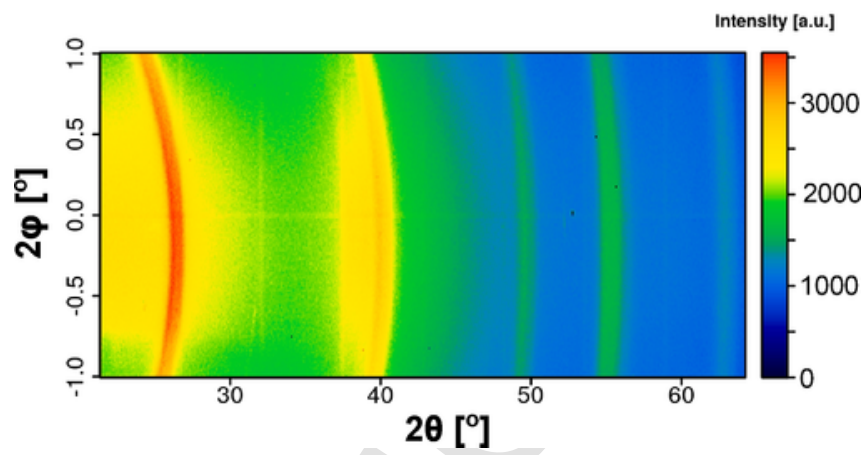

Fig. 7. GIWAXS $2 \mathrm{D}$ image from a $\mathrm{TiO}_{2}$ nanotube array sample NT-60V-60 (calcined). Angle of grazing incidence $\alpha_{i}=\alpha_{c}+1.0^{\circ}$. 20 scale values corresponds to specular plane cut values $(2 \varphi=0)$.

respectively. Presented 2D images (intensity map) as a results of GISAXS experiments were used to make $1 \mathrm{D}$ cross-sections for quantitative analysis. The dark horizontal and vertical lines are related to detector dead areas (pixels) between the CMOS modules. The GISAXS intensity is attenuated in the specular plane $\left(\mathrm{Q}_{\mathrm{y}}=0\right)$ by a partially transparent $\mathrm{Al}$ stripe positioned inside the vacuum chamber in order to avoid detector damage by the very high intensity of the directly reflected X-ray beam.

For that value of the angle of incidence X-ray beam not penetrate deep below sample surface (only $10-20 \mathrm{~nm}$ ) and GISAXS signal contains information only about sample surface morphology (Fig. 9a and c). For higher values of angle of incidence (Fig. 9b and d) beam penetrate much deeper below sample surface and provide information from larger volume below the sample surface.

For the Ti substrate sample (9a), which has a flat surface, the GISAXS maximum is well defined with wide Yoneda wings in the direction parallel to sample surface [17]. Diffuse scattering is concentrated close to specular plane $\left(\mathrm{Q}_{\mathrm{y}}=0 \mathrm{~nm}^{-1}\right)$. At the Yoneda maximum line $\left(\mathrm{Q}_{\mathrm{z}}=\right.$ const. $)$ a broad maximum can be observed at the position $Q_{y}=0.75 \mathrm{~nm}^{-1}$ which corresponds to objects 7-10 nm in size and can be related to Ti nanocrystals observed also in SEM images (Fig. 4f). As-deposited $\mathrm{Ti}$ samples are homogenous in depth and there is no additional contribution to GISAXS signal for larger value of angle of incidence.

For anodized samples, the GISAXS images (c and d) consists of the intensity contribution from the Ti substrate and the anodized circular area $\left(\mathrm{TiO}_{2} \mathrm{NT}\right.$ array) because the incident X-ray beam footprint on the sample surface is very long. Position of Yoneda maxima for Ti (Fig. 9a) is positioned at significantly higher $\mathrm{Q}_{\mathrm{y}}$ value then for $\mathrm{TiO}_{2} \mathrm{NT}$ sample (Fig. 9c). This is a direct consequence of high porosity (lower electron density) of $\mathrm{TiO}_{2}$ NT sample.

For quantitative analysis $1 \mathrm{D}$ cuts are done in direction normal to sample surface $\left(\mathrm{Q}_{\mathrm{y}}=\right.$ const.) and direction parallel to sample surface $\left(\mathrm{Q}_{\mathrm{z}}=\right.$ const.).

Intensity cuts normal to the sample surface $\left(\mathrm{Q}_{\mathrm{y}}=\right.$ const.) close to the specular plane $\left(Q_{y}=0\right)$ and outside of the beam-stop area, are used for critical angle determination (Fig. 10). Two Yoneda maxima are observed for $\mathrm{TiO}_{2}$ NT sample (Figs. 9d and 10b). The lower one is related to the NT etched area and the higher one to the $\mathrm{ZnO}$ substrate or Ti surrounding.

The position of the Yoneda maxima and the specular (directly reflected) maxima were used for the index of refraction calculation and the density estimation [18]:

$\theta_{\text {Yoneda }}=\alpha_{i}+\alpha_{c}$

$\alpha_{c} \approx \sqrt{2 \delta}$

$\delta=\frac{r_{e l}}{2 \pi} \lambda^{2} \rho_{e}$

where $\alpha_{i}$ is the angle of grazing incidence, $\alpha_{c}$ is the critical angle for total external reflection and $\delta$ is the real part of the index of refraction $n=1-\delta+i \beta, r_{e l}$ is the classical radius of the electron, $\lambda$ the wavelength and $\rho_{e}$ the electron density.

In Fig. 11 are compared 1D cuts in the direction normal to the sample surface slightly outside the beamstop region $\left(Q_{y}=-0.144 \mathrm{~nm}^{-1}\right.$ ) in order to avoid the beamstop area where the scattered intensity is attenuated. The sample with $\mathrm{TiO}_{2}$ nanotubes surrounded by substrate area shows two Yoneda maxima (critical angles for total external reflection) corresponding to two materials with different electron densities. The lower critical angle $\alpha_{C}=0.174^{\circ}$ corresponds to the porous $\mathrm{TiO}_{2}$ NT material and the higher one $\alpha_{C}=0.288^{\circ}$ corresponds to the surrounding compact dense Ti area. This value is in rather good agreement with the theoretically calculated values for Ti bulk material $(\rho=4.54$ ) $\alpha_{C}=0.299^{\circ}$ and $\mathrm{TiO}_{2}$ bulk material $(\rho=4.26) \alpha_{C}=0.295^{\circ}$. Somewhat lower values of $\alpha_{c}$ than theoretical values obtained for Ti can be explained by oxidation of Ti surface.

For the lowest used grazing angle of incidence, only one Yoneda maximum can be observed because that angle of grazing incidence is lower than the critical angle for titanium (Fig. 10b). The region around the Yoneda maximum was fitted to the well-known equation including the Fresnel transmission coefficients, and the critical angle was calculated (Eq. A6 in appendix).

The obtained critical angle for total external reflection from the $\mathrm{TiO}_{2}$ nanotube array area is much lower than the characteristic 


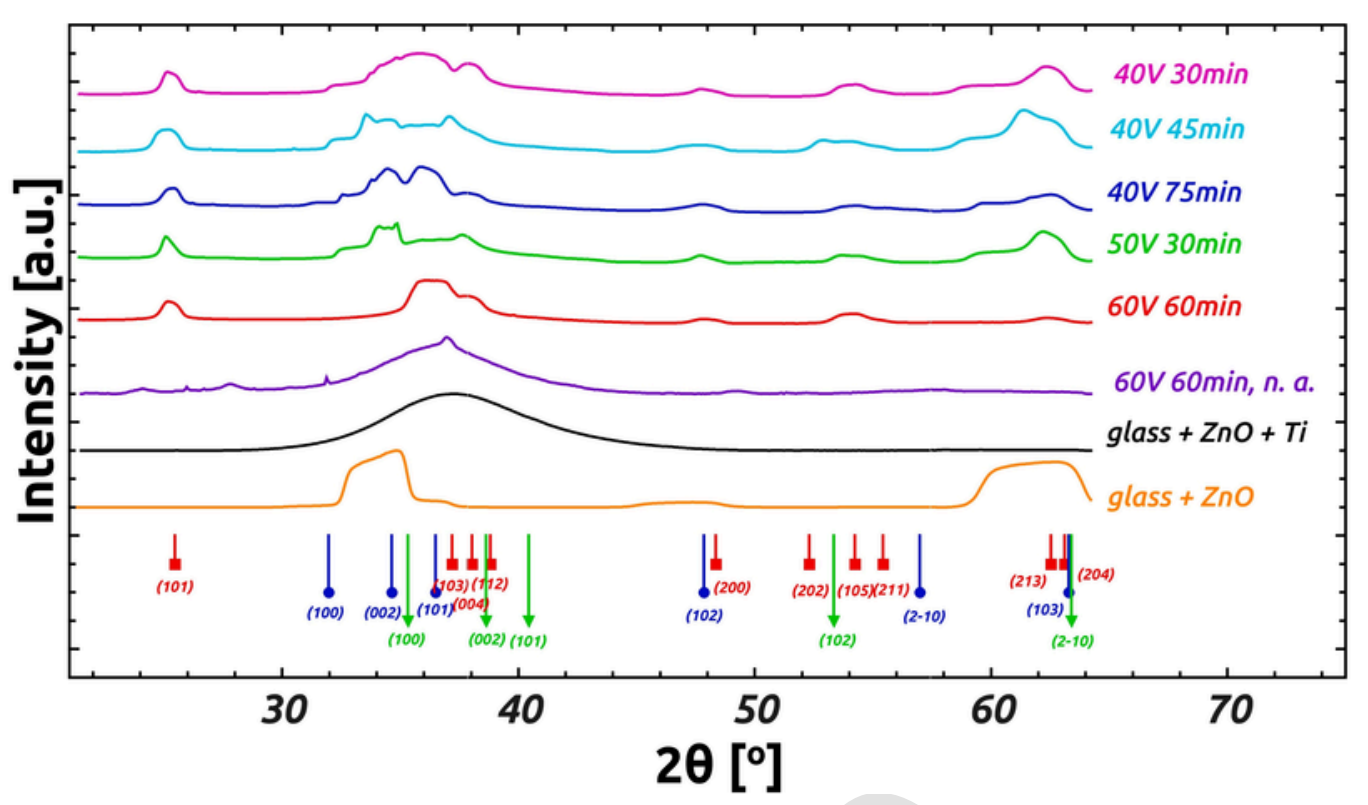

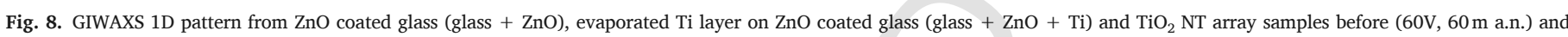

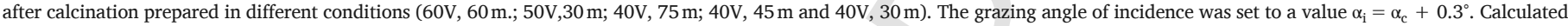
position of XRD peaks using d-spacing for bulk material are marked (red rectangles; $\mathrm{TiO}_{2}$ anatase, blue circles: $\mathrm{ZnO}$ zincite and green triangles: $\mathrm{Ti}$ ).

values for $\mathrm{Ti}$ and $\mathrm{TiO}_{2}$ bulk material. The reason is that the effective density of the $\mathrm{TiO}_{2}$ nanotube array samples is decreased due to the empty spaces between and inside the single tubes.

The porosity was calculated from the ratio of the critical angles for total external reflection for bulk $\mathrm{TiO}_{2}$ material and the $\mathrm{TiO}_{2}$ nanotube array layer using the equation [19]:

$$
\begin{aligned}
P & =\frac{\rho_{\text {bulk }}-\rho_{\text {nanotubes }}}{\rho_{\text {bulk }}} \\
& =1-\frac{\rho_{\text {nanotubes }}}{\rho_{\text {bulk }}} \\
& =1-\left[\frac{\alpha_{c \text { (nanotubes })}}{\alpha_{c(\text { bulk })}}\right]^{2}
\end{aligned}
$$

The obtained porosity values were in the range $30-40 \%$ for all analysed samples.

For the anodized samples, 1D cuts taken parallel to the sample surface at the Yoneda maximum position show maxima related to the ordered array of nanotubes produced by anodization (Fig. 11).

The Yoneda maximum vanishes for the angle of grazing incidence significantly larger than the critical angle for total external reflection (Fig. 9d). A larger angle of incidence means a larger penetration depth below the sample surface and thus an additional GISAXS scattering contribution from the bottom part of the nanotubes which is not perfectly arranged with the top part.

1D cuts in the direction parallel to sample surface at the position of Yoneda maximum are used for quantitative analysis of nanotubes lateral dimensions (inner and outer diameter). In Fig. 11 are presented $1 \mathrm{D}$ cuts for selected $\mathrm{TiO}_{2}$ NT samples prepared using different anodization voltage. Position of observed peaks is related to nanotubes dimensions. Peaks are very weak because of variation in nanotube diameter, wall thickness and nanotube inclination (presented Fig. 5, SEM side view). For a higher angle of incidence, peaks are not anymore observed because larger sample volume below the sample surface contribute to GISAXS signal and variation in nanotubes diameter and inclination are more pronounced.

To simulate GISAXS pattern from $\mathrm{TiO}_{2}$ nanotubes array samples a theoretical model was made in the framework of the Distorted Wave Born Approximation (Eq. (A4) in the Appendix). This model is based on $1 \mu \mathrm{m}$ long vertically aligned core-shell cylinders (Eq (A3), A1 in the Appendix). The inner empty cylinder and the shell with a refraction index of equal to that from bulk $\mathrm{TiO}_{2}$ were obtained from Ref. [20]. A Gaussian distribution was used for the description of the inner cylinder diameter and the shell thickness with two free parameters: mean value and standard deviation (Eq (A5) in Appendix).

The calculations were done using the BornAgain software [21] with a custom written a python script. A rectangular detector with correction for the finite pixel size of the Pilatus detector was used in the calculations. Four parameters were selected for variation and optimization: mean value and standard deviation for the inner cylinder radius, and mean value and standard deviation for the shell thickness.

During the numerical calculation ensembles of 50-100 nanotubes with different inner radii and shell thicknesses were generated by using bivariate Gaussian distributions. The Gaussian distribution parameters selection was restricted to an interval $\pm 2 \sigma$ around the mean value.

To avoid time-consuming numerical calculations during the $1 \mathrm{D}$ cuts simulation a variation of the nanotubes length and inclination was neglected. Examples of fits obtained in that way are shown in Fig. 11.

The values for the inner and outer radii obtained from the GISAXS experiments are in good agreement with the SEM results (Fig. 12). However, GISAXS gives systematically larger values for the outer nanotube radius. Side view of nanotubes (Fig. 5) shows that nanotubes have a smaller diameter at the top (close to sample surface) which is seen by SEM. Opposite to that, GISAXS gives information averaged over larger volume below sample surface (depending on the angle of incidence) where nanotube diameter is increased.

\section{Conclusion}

Vertically aligned $\mathrm{TiO}_{2}$ nanotube arrays were successfully prepared by anodization of electron beam evaporated titanium thin films. SEM and simultaneous GISWAXS were applied for the structural analysis. It was shown that ordered vertically aligned nanotube arrays could be obtained, with single nanotube outer diameters in the range of $50-100 \mathrm{~nm}$, inner diameters of $15-40 \mathrm{~nm}$ and an average layer porosity of $30-40 \%$. A model was developed for the simulation of the GISAXS pattern obtained from $\mathrm{TiO}_{2}$ nanotubes arrays. The simulation results are in good agreement with SEM data which verifies the way of analysis 

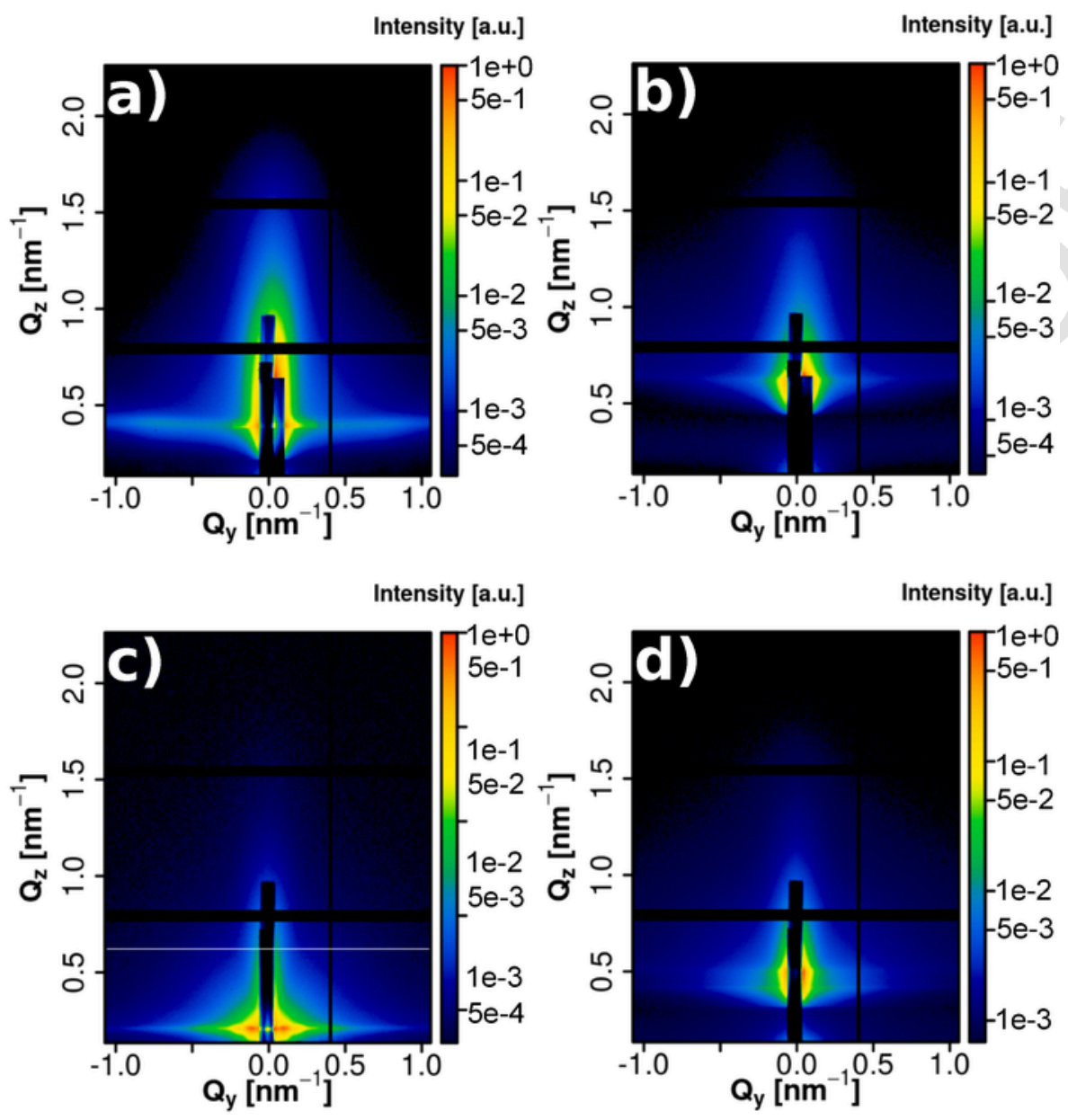

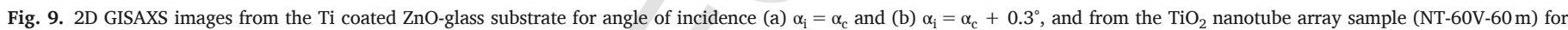
angle of incidence (c) $\alpha_{i}=\alpha_{c}$ and (d) $\alpha_{i}=\alpha_{c}+0.3^{\circ}$.
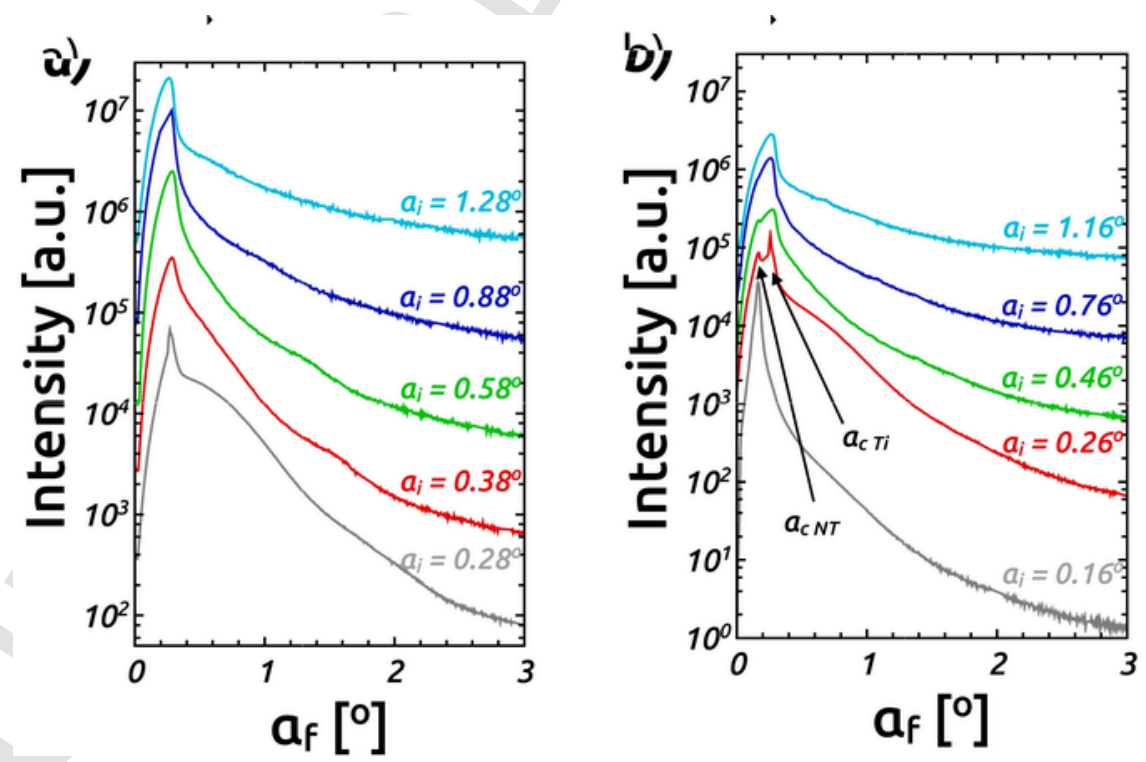

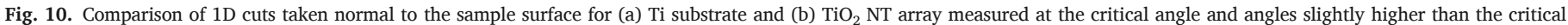
angle. The position of Yoneda maxima for the $\mathrm{Ti}$ surrounding and $\mathrm{TiO}_{2}$ nanotubes are annotated by arrows. 


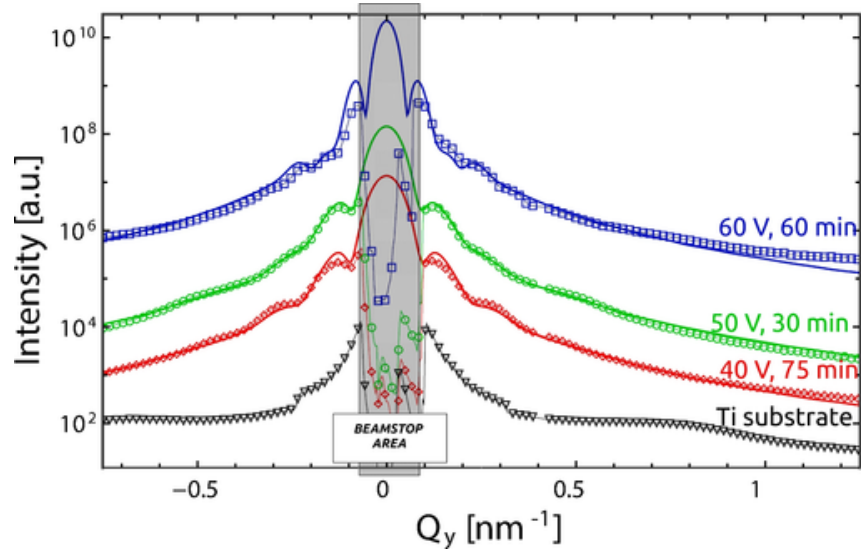

Fig. 11. GISAXS 1D cuts in direction parallel to the sample surface taken at the Yoneda maximum measured at the critical angle for total external reflection. Anodization voltage and time are denoted above 1D cuts. Experimental data are presented with the symbols while results of the BornAgain theoretical simulation are presented with full thick lines. Detector area shielded by $\mathrm{Al}$ beamstop is presented by gray rectangle ("Beamstop area").
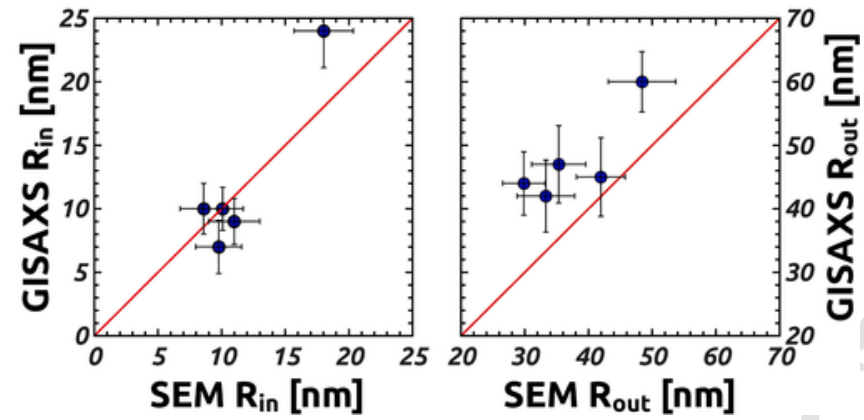

Fig. 12. Comparison of inner and outer radius of the nanotubes obtained by modelling of GISAXS 1D cuts and SEM images analysis. Error bars present the standard deviation of the gaussian distribution for GISAXS and SEM). Red lines present the unity slope (equal values for GISAXS and SEM).

of GISWAXS spectra and enables the use of this experimental technique as a fast and efficient tool for the NT arrays study. Compared to SEM, GISAXS can provide detailed structural information for a wide range of experimental conditions, especially for in-situ studies.

\section{Acknowledgements}

We acknowledge the European Social Found (project: Titanium dioxide nanostructures for photovoltaics: professional development of young researchers/postdoctorands) and Croatian science foundation (projects: "Zinc Oxide and Titanium Dioxide Nanocomposites for Photovoltaic Application" - ZOTONanoPhotovolt and "Nanocomposites comprising perovskites for photovoltaics, photo-catalysis and sensing" - 2014-09-9419) for financial support.

\section{Appendix.}

The cylinder form factor is [22]:

$F=2 \pi R^{2} H \operatorname{sinc}\left(q_{z} \frac{H}{2}\right) \exp \left(i q_{z} \frac{H}{2}\right) \frac{J_{1}\left(q_{\|} R\right)}{q_{\|} R}$

where $\mathrm{R}$ is the cylinder radius, $\mathrm{H}$ the cylinder height, qx, qy, qz, components of the scattering vector, and

$q_{\|}=\sqrt{q_{x}^{2}+q_{y}^{2}}$ [22]

The core shell (hollow) particle (shape) form factor is calculated as

$F_{c s}(\vec{q})=F_{c o}+\tau\left(F_{s h}-F_{c o}\right)$

where $F_{c o}$ is the form factor of the inner cylinder with radius $R_{\text {inner }}$ and index of refraction $n_{c o}$ (in our calculations equal to 1 ), $F_{s h}$ is the form factor of the outer cylinder with radius $R_{\text {outer }}$ and index of refraction $n_{s h}$ (in our case the value for bulk $\mathrm{TiO}_{2}$ ), and. $\tau=\frac{1-n_{s h}^{2}}{1-n_{c o}^{2}}$

Calculations are done in the framework of the Distorted Wave Born Approximation (DWBA). In the DWBA the particle form factor is calculated as the sum of four terms involved in the scattering process associated to different scattering events [22]:

$F_{\text {tot }}\left(q_{\|}, k_{z}^{i}, k_{z}^{f}\right)=F\left(q_{\|}, k_{z}^{f}-k_{z}^{i}\right)+R_{F}\left(\alpha_{i}\right) F\left(q_{\|}, k_{z}^{f}+k_{z}^{i}\right)+R_{F}\left(\alpha_{f}\right) F\left(q_{\|}\right.$

where $k_{z}^{i}$ and $k_{z}^{i}$ are the $\mathrm{z}$ components (normal to the sample surface) of the incident and scattered wave vectors, $R_{F}$ is the Fresnel refraction factor, $\alpha_{i}$ the angle of grazing incidence, $\alpha_{f}$ the scattering angle. The total form factor is calculated as 2D integral of the core-shell cylinder form factors with normal distributed inner radius and shell thickness $\left(d_{\text {shell }}=R_{\text {out }}-R_{\text {in }}\right)$. To calculate a $2 \mathrm{D}$ integral it is generated a $2 \mathrm{D}$ equally distributed grid in intervals $\left(\begin{array}{c}R_{\text {in } m}-2 \sigma_{R_{i n}}, R_{\text {in } m}-2 \sigma_{R_{\text {in }}} ; d_{\text {shell } m} \\ -2 \sigma_{d_{s h l}}, d_{\text {shell } m}-2 \sigma_{d_{\text {shl }}}\end{array}\right.$ ). For each mesh in the grid is calculated a weighting factor for the form factor scaling as bivariate normal distribution:

$W\left(R_{\text {in }}, d_{\text {shell }}\right)$

$=\frac{1}{2 \pi \sigma_{R_{\text {in }}} \sigma_{\mathrm{d}_{\text {shell }}}} e^{-\frac{1}{2}\left[\left(\frac{R_{\text {in }}-R_{\text {in } m}}{\sigma_{R_{\text {in }}}}\right)^{2}\left(\frac{d_{\text {shell }}-d_{\text {shell } m}}{\sigma_{d_{\text {shell }}}}\right)^{2}\right]}$

where $R_{i n}, \sigma_{R_{i n}}, d_{\text {shell }}, \sigma_{d_{\text {shell }}}$ are parameters (mean value and standard deviation) of the inner radius and shell thickness normal distribution.

The scattered intensity below and around the Yoneda maximum can be described by:

$I(\vec{q})=\mathrm{A}\left|t\left(\overrightarrow{k_{i}}\right) t\left(\overrightarrow{k_{f}}\right)\right|^{2} \mathrm{~F}(\overrightarrow{\mathrm{q}})$

where A is constant, $F(\vec{q})$ is the form factor, and $t\left(\vec{k}_{i}\right)$ and $t\left(\vec{k}_{f}\right)$ are Fresnel factors for the refraction of the incident and scattered wave vectors:

$t=\frac{2 k_{i, z}}{k_{i, z}+k_{t, z}}=\frac{2 \sin \alpha_{i}}{\sin \alpha_{i}+\sqrt{n^{2}-\cos ^{2} \alpha_{i}}}$

where $\alpha_{i}$ is the angle of incidence and $\mathrm{n}$ is the complex index of refraction.

X-ray attenuation length $\Lambda$ is defined as the depth into the material measured along the surface normal where the intensity of x-rays falls to 1/e of its value at the surface [23]:

$\Lambda=\frac{\lambda}{2 \sqrt{2} \pi}\left\{\sqrt{\sqrt{\left(\alpha_{i}^{2}-\alpha_{c}^{2}\right)^{2}+4 \beta^{2}}-\alpha_{i}^{2}+\alpha_{c}^{2}}\right\}^{-1}$

where $\alpha_{i}$ is the angle of grazing incidence, $\alpha_{c}$ is the critical angle for total external reflection and $\beta$ is the imaginary part of the index of refraction $n=1-\delta+i \beta$ and $\lambda$ the wavelength.

\section{References}

[1] M. Grätzel, Dye-sensitized solar cells, J. Photochem. Photobiol. C Photochem. Rev. 4 (2003) 145-153, https://doi.org/10.1016/S1389-5567(03)00026-1. 
[2] M. Ni, M.K.H. Leung, D.Y.C. Leung, K. Sumathy, A review and recent developments in photocatalytic water-splitting using $\mathrm{TiO} 2$ for hydrogen production, Renew. Sustain. Energy Rev. 11 (2007) 401-425, https://doi.org/10.1016/j.rser. 2005.01.009.

[3] M. Paulose, K. Shankar, O.K. Varghese, G.K. Mor, B. Hardin, C.A. Grimes, Corrigendum on "Backside illuminated dye-sensitized solar cells based on titania nanotube array electrodes,", Nanotechnology 21 (2010) https://doi.org/10.1088/ 0957-4484/21/38/389801, 389801-389801.

[4] B. Tan, Y. Wu, Dye-sensitized solar cells based on anatase $\mathrm{TiO}_{2}$ nanoparticle/ nanowire composites, J. Phys. Chem. B 110 (2006) 15932-15938, https://doi.org/ 10.1021/jp063972n.

[5] V.V. Kislyuk, O.P. Dimitriev, Nanorods and nanotubes for solar cells, J. Nanosci. Nanotechnol. 8 (2008) 131-148, https://doi.org/10.1166/jnn.2008.N16.

[6] A. Ghicov, P. Schmuki, Self-ordering electrochemistry: a review on growth and functionality of TiO2 nanotubes and other self-aligned MOx structures, Chem. Commun. (2009) 2791, https://doi.org/10.1039/b822726h

[7] J.M. Macak, H. Tsuchiya, A. Ghicov, K. Yasuda, R. Hahn, S. Bauer, P. Schmuki, TiO2 nanotubes: self-organized electrochemical formation, properties and applications, Curr. Opin. Solid State Mater. Sci. 11 (2007) 3-18, https://doi.org/10.1016/ j.cossms.2007.08.004.

[8] A. Tighineanu, T. Ruff, S. Albu, R. Hahn, P. Schmuki, Conductivity of TiO2 nanotubes: influence of annealing time and temperature, Chem. Phys. Lett. 494 (2010) 260-263, https://doi.org/10.1016/j.cplett.2010.06.022.

[9] N. Paul, J. Brumbarov, A. Paul, Y. Chen, J.-F. Moulin, P. Müller-Buschbaum, J. Kunze-Liebhäuser, R. Gilles, GISAXS and TOF-GISANS studies on surface and depth morphology of self-organized $\mathrm{TiO}_{2}$ nanotube arrays: model anode material in Li-ion batteries, J. Appl. Crystallogr. 48 (2015) 444-454, https://doi.org/10.1107/ S1600576715002204.

[10] V. Mandić, M. Plodinec, I. Kereković, K. Juraić, V. Janicki, D. Gracin, A. Gajović, A. Moguš-Milanković, M.G. Willinger, Tailoring anatase nanotubes for the photovoltaic device by the anodization process on behalf of microstructural features of titanium thin film, Sol. Energy Mater. Sol. Cells 168 (2017) 136-145, https://doi. org $/ 10.1016 /$ j.solmat.2017.04.028.

[11] M. Rauscher, T. Salditt, H. Spohn, Small-angle x-ray scattering under grazing incidence: the cross section in the distorted-wave Born approximation, Phys. Rev. B. 52 (1995) 16855-16863, https://doi.org/10.1103/PhysRevB.52.16855.
[12] M. Plodinec, A. Šantić, J. Zavašnik, M. Čeh, A. Gajović, Giant persistent photoconductivity in $\mathrm{BaTiO}_{3} / \mathrm{TiO}_{2}$ heterostructures, Appl. Phys. Lett. 105 (2014) 152101 https://doi.org/10.1063/1.4897999.

[13] B. Yin, Q. Qian, Z. Xiong, H. Jiang, Y. Lin, D. Feng, Growth orientation mechanism of $\mathrm{TiO}_{2}$ nanotubes fabricated by anodization, Nanotechnology 30 (2019) 155702, https://doi.org/10.1088/1361-6528/aafd54.

[14] H. Amenitsch, S. Bernstorff, P. Laggner, High-flux beamline for small-angle x-ray scattering at ELETTRA, Rev. Sci. Instrum. 66 (1995) 1624-1626, https://doi.org/ $10.1063 / 1.1145864$.

[15] M. Plodinec, I. Grčić, M.G. Willinger, A. Hammud, X. Huang, I. Panžić, A. Gajović, Black TiO2 nanotube arrays decorated with $\mathrm{Ag}$ nanoparticles for enhanced visible-light photocatalytic oxidation of salicylic acid, J. Alloy. Comp. 776 (2019) 883-896, https://doi.org/10.1016/j.jallcom.2018.10.248.

[16] J. Ilavsky, Nika Package for 2D -> 1D SAS Data Reduction, 2018, Nika package for 2D - > 1D SAS data reduction.

[17] Y. Yoneda, Anomalous surface reflection of X rays, Phys. Rev. 131 (1963) 2010-2013, https://doi.org/10.1103/PhysRev.131.2010

[18] U. Pietsch, V. Holý, T. Baumbach, High-Resolution X-Ray Scattering, Springer New York, New York, NY, 2004https://doi.org/10.1007/978-1-4757-4050-9.

[19] S. Dourdain, A. Mehdi, J.F. Bardeau, A. Gibaud, Determination of porosity of mesoporous silica thin films by quantitative X-ray reflectivity analysis and GISAXS Thin Solid Films 495 (2006) 205-209, https://doi.org/10.1016/j.tsf.2005.08.356.

[20] B.L. Henke, E.M. Gullikson, J.C. Davis, X-ray interactions: photoabsorption, scattering, transmission, and reflection at $\mathrm{E}=50-30,000 \mathrm{eV}, \mathrm{Z}=1-92$, Atomic Data Nucl. Data Tables 54 (1993) 181-342, https://doi.org/10.1006/adnd.1993.1013.

[21] C. Durniak, M. Ganeva, G. Pospelov, W. Van Herck, J. Wuttke, BornAgain — Software for Simulating and Fitting X-Ray and Neutron Small-Angle Scattering at Grazing Incidence, Version 1.10, 2017 http://www.bornagainproject.org http://www. bornagainproject.org.

[22] R. Lazzari, IsGISAXS : a Tool for Analyzing Grazing Incidence Small Angle X-Ray Scattering Analysis from Nanostructures, Version 2.6, 2015 http://www.insp. jussieu.fr/oxydes/IsGISAXS/isgisaxs.htm.

[23] J. Als-Nielsen, D. McMorrow, Elements of Modern X-Ray Physics, second ed., Wiley, Hoboken, 2011. 